\title{
Equilibrium control of nonlinear verticum-type systems, applied to
}

\section{integrated pest control}

\author{
S. Molnár ${ }^{1}$, M. Gámez ${ }^{2 *}$, I. López ${ }^{2}$ and T. Cabello ${ }^{3}$ \\ ${ }^{1}$ Institute of Mathematics and Informatics, Szent István University, \\ Páter K. u. 1., H-2103 Godollo, Hungary, (Molnar.Sandor@gek.szie.hu) \\ ${ }^{2}$ Department of Statistics and Applied Mathematics, University of Almería, \\ La Cañada de San Urbano 04120 Almería, Spain (mgamez@ual.es, milopez@ual.es) \\ ${ }^{3}$ Department of Applied Biology, University of Almería, \\ La Cañada de San Urbano 04120 Almería, Spain (tcabello@ual.es)
}

\begin{abstract}
Linear verticum-type control and observation systems have been introduced for modelling certain industrial systems, consisting of subsystems, vertically connected by certain state variables. Recently the concept of verticum-type observation systems and the corresponding observability condition have been extended by the authors to the nonlinear case. In the present paper the general concept of a nonlinear verticum-type control system is introduced, and a sufficient condition for local controllability to equilibrium is obtained. In addition to a usual linearization, the basic idea is a decomposition of the control of the whole system into the control of the subsystems. Starting from the integrated pest control model of Rafikov and E.H. Limeira (2012) a nonlinear verticum-type model has been set up an equilibrium control is obtained. Furthermore, a corresponding bioeconomical problem is solved minimizing the total cost of integrated pest control (combining chemical control with a biological one).
\end{abstract}

Keywords: verticum-type system, nonlinear control system, integrated pest control.

\section{Introduction}

Verticum-type systems have been introduced by Molnár (1989) for modelling certain industrial systems. These systems, are hierarchically composed of linear subsystems such that a part of the state variables of each subsystem affect the dynamics of the next subsystem. In Molnár (1989), for continuous-time linear systems, necessary and sufficient conditions for observability and controllability of such systems were obtained. Other systems-theoretical properties of such systems were studied e.g. in Molnár (1993), Molnár and Szigeti (1994).

Recently, apart from industrial systems, verticum-type system models found applications in population ecology. In fact, population interactions are typically nonlinear, but in Gámez et al. (2010) an ecological interaction chain of the type resource - producer - primary user - secondary consumer turned out to have a verticum-like structure which admitted to reduce the monitoring (observability) problem to a linearized version of the original model. In Molnár et al. (2012), the concept of a

${ }^{*}$ Corresponding author 
nonlinear verticum-type observation system has been introduced, and a decomposition of the sufficient condition for observability of such systems have been proved.

The possible applications to dynamic models of population biology is a strong motivation for the introduction and study of nonlinear verticum-type control systems which is the aim the present paper. The application of mathematical systems theory to monitoring and control of population systems was initialized in Varga (1989) and (1992), providing sufficient conditions for local controllability and observability of nonlinear systems with invariant manifold to frequency-dependent population models. For further results on similar population systems, see also Scarelli and Varga (2002), López et al. (2004) and Varga (2008a). Systems-theoretical study of density-dependent multi-species population models can be found e.g. in Varga et al. (2002), (2003), Shamandy (2005), López et al. (2007a,b), Gámez et al. (2009, 2011, 2012), For a general review on the application of mathematical systems theory in population biology, see Varga (2008b), a recent update of this survey is Gámez (2011).

In the present paper the concept of a nonlinear verticum-type system is introduced and a sufficient condition for local controllability to equilibrium is obtained. The application of the obtained results are then illustrated on a model of integrated pest control, based on the model of Rafikov and Limeira (2012) concerning biological control of the Sugarcane Borer (Diatraea sacharalis). Integrated control means, that a combination of a biological agent and a chemical pesticide is applied.

The paper is organized as follows. In section 2, we introduce the concept of a nonlinear verticum-type system, and in terms of the linearization of the single subsystems, a Kalman-type sufficient condition is obtained for the controllability of the whole system. Section 3 is devoted to the study of a population system where the theoretical results of the previous section are applied. First, we set up a multistage population dynamics model for the interaction of sugar cane borer with its egg parasitoid. In 3.1 and 3.2, the existence of a positive equilibrium and its asymptotic stability are proved. In subsections the 3.3 and 3.4, supposing that the pest is controlled by the release of parasitoid and by the application of a pesticide, we obtain a nonlinear verticum-type control system, and apart from proving controllability of the system to equilibrium, the corresponding equilibrium control is also constructed. Finally, an optimal equilibrium control is obtained that minimizes the total costs of simultaneous application of biological and chemical control.

\section{Controllability of nonlinear verticum-type systems into equilibrium}

Given $k, n_{i}, r_{i} \in N, i \in \overline{0, k}, n:=\sum_{i=0}^{k} n_{i}, r:=\sum_{i=0}^{k} r_{i}$ and $F: R^{n} \times R^{r} \rightarrow R^{n}$, a continuously differentiable function.

For a control $\quad u^{*}:=\left(u_{0}^{*}, u_{1}^{*}, \ldots, u_{k}^{*}\right) \in R^{r} \quad$ with $\quad u_{i}^{*} \in R^{r_{i}}, i \in \overline{0, k}$, let $x^{*}:=\left(x_{0}^{*}, x_{1}^{*}, \ldots, x_{k}^{*}\right) \in R^{n}$ with $x_{i}^{*} \in R^{n_{i}}(i \in \overline{0, k})$ such that $F\left(x^{*}, u^{*}\right)=0$.

Let us fix a time interval [0,T], and for each $\varepsilon>0$ define the class of $\varepsilon$-small controls $U_{\varepsilon}[0, T]$ as in the Appendix. 
Consider the nonlinear control system

$$
\dot{x}_{0}=F_{0}\left(x_{0}, u_{0}^{*}+u_{0}\right) ; \quad F_{0}: R^{n_{0}} \times R^{r_{0}} \rightarrow R^{n_{0}},
$$

and for all $i \in \overline{1, k}$

$$
\dot{x}_{i}=F_{i}\left(x_{i}, x_{i-1}, u_{i}^{*}+u_{i}\right) ; \quad F_{i}: R^{n_{i}} \times R^{n_{i-1}} \times R^{r_{i}} \rightarrow R^{n_{i}},
$$

and define

$$
F\left(x, u^{*}+u\right):=\left(F_{0}\left(x_{0}, u_{0}^{*}+u_{0}\right), F_{1}\left(x_{1}, x_{0}, u_{1}^{*}+u_{1}\right), \ldots, F_{k}\left(x_{k}, x_{k-1}, u_{k}^{*}+u_{k}\right)\right)
$$

\section{Definition 2.1.}

$$
\dot{x}=F\left(x, u^{*}+u\right)
$$

is said to be a (nonlinear) verticum-type control system with subsystems $\left(V_{i}\right)(i \in \overline{0, k})$.

Remark 2.1. Equations $\left(V_{i}\right)$ do not define a standard control system in this setting, because of the presence of the "exogenous" variable $x_{i-1}$ connecting it to equation $\left(V_{i-1}\right)(i \in \overline{1, k})$.

Remark 2.2. From Theorem A.1 of the Appendix we obtain that there exists $\varepsilon_{0} \in R^{+}$ such that for all $u \in U_{\varepsilon_{0}}[0, T]$ and $x^{0} \in R^{n}$ with $\left\|x^{0}-x^{*}\right\|<\varepsilon_{0}$ the initial value problem

$$
\begin{aligned}
& \dot{x}(t)=F\left(x(t), u^{*}+u(t)\right) \quad(\text { for a.e. } t \in[0, T]) \\
& x(0)=x^{0}
\end{aligned}
$$

has a unique solution. In what follows $T>0$ will be considered fixed and concerning controllability, the reference to it will be often suppressed.

To study controllability of system $(V)$, let us linearize systems $\left(V_{0}\right)$, at respective equilibria $\left(x_{0}^{*}, u_{0}^{*}\right)$, obtaining the linearized systems

$$
\dot{x}_{0}=A_{00} x_{0}+B_{0} u_{0},
$$

where

$$
A_{00}=\frac{\partial F_{0}}{\partial x_{0}}\left(x_{0}^{*}, u_{0}^{*}\right), \quad B_{0}=\frac{\partial F_{0}}{\partial u_{0}}\left(x_{0}^{*}, u_{0}^{*}\right)
$$

and for all $i \in \overline{1, k}$, substituting $x_{i-1}$ in $\left(V_{i}\right)$ with its equilibrium value $x_{i-1}^{*}$, we similarly linearize $\left(V_{i}\right)$ with respect to variables $\left(x_{i}, u_{i}\right)$, at the corresresponding equilibrium $\left(x_{i}^{*}, u_{i}^{*}\right)$, obtaining the linearized systems

$$
\dot{x}_{i}=A_{i i} x_{i}+B_{i} u_{i},
$$

with $A_{i i}=\frac{\partial F_{i}}{\partial x_{i}}\left(x_{i}^{*}, x_{i-1}^{*}, u_{i}^{*}\right) ; \quad B_{i}=\frac{\partial F_{i}}{\partial u_{i}}\left(x_{i}^{*}, x_{i-1}^{*}, u_{i}^{*}\right) \quad(i \in \overline{1, k})$. 
Now we define the matrices $A \in R^{n \times n}, B \in R^{n \times r}$ as follows:

$$
A=\left(\begin{array}{ccccccc}
A_{00} & 0 & 0 & \ldots & 0 & \ldots & 0 \\
A_{10} & A_{11} & 0 & \ldots & 0 & \ldots & 0 \\
0 & A_{21} & A_{22} & \ldots & 0 & \ldots & 0 \\
\cdot & . & . & \ldots & . & \ldots & . \\
. & . & . & \ldots & . & \ldots & . \\
. & . & . & \ldots & . & \ldots & . \\
0 & 0 & 0 & \ldots & . & A_{k-1, k-1} & 0 \\
0 & 0 & 0 & \ldots & . & A_{k, k-1} & A_{k, k}
\end{array}\right),
$$

where

$$
\begin{aligned}
& A_{i i-1}=\frac{\partial F_{i}}{\partial x_{i-1}}\left(x_{i}^{*}, x_{i-1}^{*}, u_{i}^{*}\right), \quad(i \in \overline{1, k}) . \\
& B=\left(\begin{array}{ccccc}
B_{0} & & & & 0 \\
& B_{1} & & & \\
& & \cdot & & \\
& & & \cdot & \\
0 & & & & B_{k}
\end{array}\right) \text {, }
\end{aligned}
$$

obtaining linear control system

$$
\dot{x}=A x+B u
$$

of verticum type (see Molnár, 1989).

We recall a sufficient condition for controllability of linear verticum-type systems.

Theorem 2.1. (Molnár, 1989) Suppose that

$$
\operatorname{rank}\left[\begin{array}{c}
B_{i} \\
A_{i i} B_{i} \\
\vdots \\
A_{i i}^{n_{i i}-1} B_{i}
\end{array}\right]=n_{i} \quad(i \in \overline{0, k}) .
$$

Then the linear verticum-type system $(L V)$ is controllable.

Suppose that for each $i \in \overline{0, k}$

$$
\operatorname{rank}\left[\begin{array}{c}
B_{i} \\
A_{i i} B_{i} \\
\vdots \\
A_{i i}^{n_{i}-1} B_{i}
\end{array}\right]=n_{i}
$$

then by Theorem 2.1 the verticum-type system $(L V)$ is controllable. 
Hence, the linearization of the control system $(V)$ is controllable. Therefore, by Kalman's theorem on controllability of linear systems (see Lee and Markus, 1969), the rank condition (A.3) of the Appendix is fulfilled, implying local controllability of system $(V)$ to equilibrium $x^{*}$.

The above reasoning can be summarized in the following sufficient condition for local controllability of nonlinear verticum-type systems:

Theorem 2.2. If

$$
\operatorname{rank}\left[\begin{array}{c}
B_{i} \\
A_{i i} B_{i} \\
\vdots \\
A_{i i}^{n_{i}-1} B_{i}
\end{array}\right]=n_{i} \quad(i \in \overline{0, k}) \text {, }
$$

then control system $(V)$ is controllable near its equilibrium $x^{*}$.

In what follows the above result will be applied to the analysis of a control system modeling integrated pest control.

\section{Application to the model for the integrated control sugarcane borel (Diatraea saccharalis) by an egg parasitoid (Trichogramma galloi), combined with chemical control}

Consider the mathematical model of interaction between the sugarcane borer (Diatraea saccharalis) and its egg parasitoid Trichogramma galloi which consist of three differential equations, proposed in Rafikov and Limeira (2012), from which we have added it one more differential equation:

$$
\begin{aligned}
& \dot{x}_{1}=r\left(1-\frac{x_{1}}{K}\right) x_{1}-m_{1} x_{1}-n_{1} x_{1}-\beta x_{1} x_{2} \\
& \dot{x}_{2}=\beta x_{1} x_{2}-m_{2} x_{2}-n_{2} x_{2} \\
& \dot{x}_{3}=n_{1} x_{1}-m_{3} x_{3}-n_{3} x_{3} \\
& \dot{x}_{4}=n_{3} x_{3}-m_{4} x_{4}-n_{4} x_{4}
\end{aligned}
$$

where

$x_{1}=$ egg density of the sugarcane borer,

$x_{2}=$ density of eggs parasitized by Trichogramma galloi,

$x_{3}=$ small larvae density of the sugarcane borer,

$x_{4}=$ large larvae density of the sugarcane borer,

$r=$ net reproduction rate,

$K=$ carrying capacity the environment,

$m_{1}, m_{2}, m_{3}, m_{4}=$ mortality rates of egg, parasitized egg, small larvae and large larvae populations, respectively, 


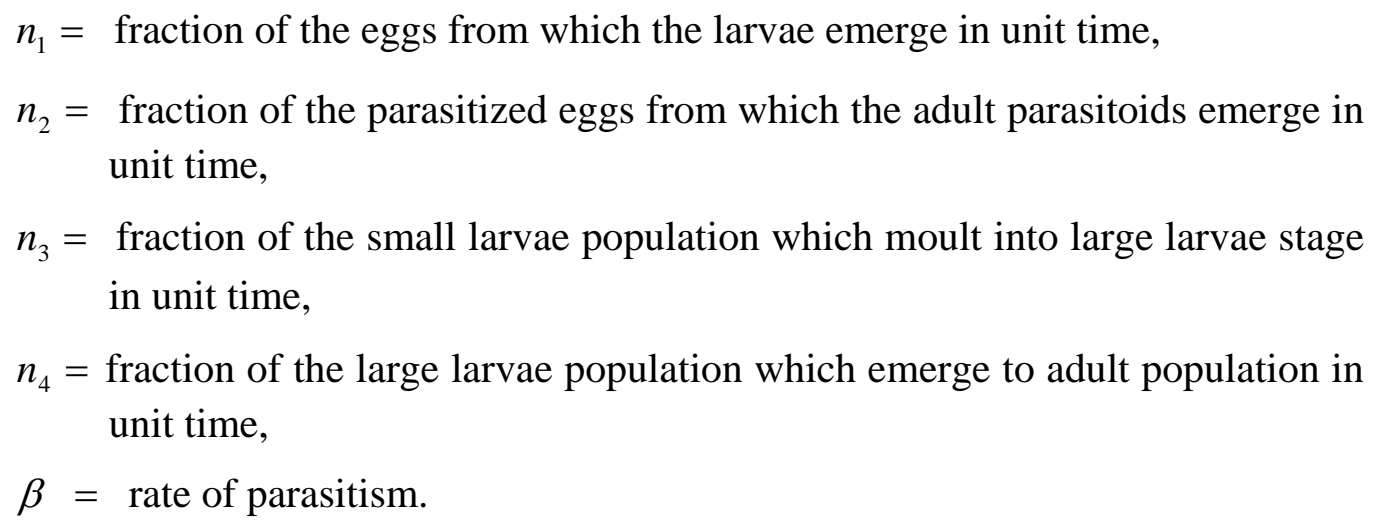

In the following two subsections we investigate the stable coexistence in the model.

\subsection{Existence of a positive equilibrium}

First, we find a necessary and sufficient condition for the existence of a positive equilibrium of system (3.1). To this end, define functions $f_{0}: R^{2} \rightarrow R^{2}$,

$$
f_{0}\left(x_{1}, x_{2}\right):=\left(\begin{array}{c}
r x_{1}\left(1-\frac{x_{1}}{K}\right)-m_{1} x_{1}-n_{1} x_{1}-\beta x_{1} x_{2} \\
\beta x_{1} x_{2}-m_{2} x_{2}-n_{2} x_{2}
\end{array}\right)
$$

and $f_{1}: R^{3} \rightarrow R^{2}$

$$
f_{1}\left(x_{1}, x_{3}, x_{4}\right):=\left(\begin{array}{c}
n_{1} x_{1}-m_{3} x_{3}-n_{3} x_{3} \\
n_{3} x_{3}-m_{4} x_{4}-n_{4} x_{4}
\end{array}\right) .
$$

Then the right-hand side of (3.1) is given by $f: R^{4} \rightarrow R^{4}$,

$$
f(x)=f\left(x_{1}, x_{2}, x_{3}, x_{4}\right):=\left[\begin{array}{c}
f_{0}\left(x_{1}, x_{2}\right) \\
f_{1}\left(x_{1}, x_{3}, x_{4}\right)
\end{array}\right] .
$$

Then a nonzero vector $x^{*} \in R^{4}$ is an equilibrium for the considered dynamics if and only if $f\left(x^{*}\right)=0$. The unique solution of this equation is easily obtained:

$$
\begin{aligned}
& x_{1}^{*}=\frac{n_{2}+m_{2}}{\beta} \\
& x_{2}^{*}=\frac{\beta K r-r\left(n_{2}+m_{2}\right)-\left(n_{1}+m_{1}\right) K \beta}{\beta} \\
& x_{3}^{*}=\frac{n_{1}\left(n_{2}+m_{2}\right)}{\beta\left(n_{3}+m_{3}\right)} \\
& x_{4}^{*}=\frac{n_{1} n_{3}\left(n_{2}+m_{2}\right)}{\beta\left(n_{3}+m_{3}\right)\left(n_{4}+m_{4}\right)}
\end{aligned}
$$

Now it is easy to see that a necessary and sufficient condition for $x^{*}>0$ is 


$$
r>n_{1}+m_{1}+\frac{r}{K \beta}\left(n_{2}+m_{2}\right) .
$$

In biological terms this condition says that fixing all parameters in (3.4), except $K$, a carrying capacity for sugarcane borer high enough implies coexistence in the system. Alternatively, rearranging (3.4) in the form

$$
r\left[K \beta-\left(n_{2}+m_{2}\right)\right]>K \beta\left(n_{1}+m_{1}\right),
$$

we obtain that, fixing all parameters in (3.4), except $r$, if the Malthus parameter of the sugarcane borer is high enough, the coexistence is also guaranteed.

The stability of coexistence is analyzed in the next subsection.

\subsection{Asymptotical stability of the equilibrium}

System (3.1) is a nonlinear verticum-type system in the sense of the Definition 2.1, composed from the following subsystems:

$$
\begin{aligned}
& \dot{x}_{1}=r x_{1}\left(1-\frac{x_{1}}{K}\right)-m_{1} x_{1}-n_{1} x_{1}-\beta x_{1} x_{2} \\
& \dot{x}_{2}=\beta x_{1} x_{2}-m_{2} x_{2}-n_{2} x_{2}
\end{aligned}
$$

and

$$
\begin{aligned}
& \dot{x}_{3}=n_{1} x_{1}-m_{3} x_{3}-n_{3} x_{3} \\
& \dot{x}_{4}=n_{3} x_{3}-m_{4} x_{4}-n_{4} x_{4}
\end{aligned}
$$

First we analyze the stability of the single subsystems. For system (3.5), we calculate the Jacobian

$$
f_{0}^{\prime}\left(x^{1}\right)=\left(\begin{array}{cc}
r-2 \frac{r}{K} x_{1}-m_{1}-n_{1}-x_{2} \beta & -\beta x_{1} \\
\beta x_{2} & 0
\end{array}\right),
$$

where $x^{1}=\left(x_{1}, x_{2}\right)$. For subsystem (3.5) at the equilibrium $x^{1 *}:=\left(x_{1}^{*}, x_{2}^{*}\right)$ we have

$$
A_{0}:=f_{0}^{\prime}\left(x^{1^{*}}\right)=\left(\begin{array}{cc}
-\frac{r}{K} x_{1}^{*} & -\beta x_{1}^{*} \\
\beta x_{2}^{*} & 0
\end{array}\right),
$$

the characteristic polynomial of which is

$$
\lambda^{2}+\frac{r}{K} x_{1}^{*} \lambda+\beta^{2} x_{1}^{*} x_{2}^{*}=0
$$

Hence, by the Routh-Hurwitz criterion we obtain the asymptotic stability of equilibrium $x^{1^{*}}$.

Repeating the same reasoning for subsystem (3.6), we have

$$
f_{1}^{\prime}\left(x_{1}^{*}, x^{2}\right)=\left(\begin{array}{cc}
-m_{3}-n_{3} & 0 \\
n_{3} & -m_{4}-n_{4}
\end{array}\right),
$$


where $x^{2}=\left(x_{3}, x_{4}\right)$. Subsystem (3.6) has a positive equilibrium $\left(x_{1}^{*}, x^{2^{*}}:=\left(x_{3}^{*}, x_{4}^{*}\right)\right)$, and the Jacobian $A_{1}:=f_{1}^{\prime}\left(x_{1}^{*}, x^{2^{*}}\right)$ has negative eigenvalues

$$
\lambda_{1}=-m_{3}-n_{3} ; \lambda_{2}=-m_{4}-n_{4},
$$

implying asymptotic stability of equilibrium $\left(x_{1}^{*}, x^{2^{*}}\right)$ of subsystem (3.6).

Now we analyze the stability of the positive equilibrium $x^{*}=\left(x_{1}^{*}, x_{2}^{*}, x_{3}^{*}, x_{4}^{*}\right)$ of system (3.1). The corresponding Jacobian is

$$
A:=f^{\prime}\left(x^{*}\right)=\left(\begin{array}{cccc}
A_{0} & 0 & 0 \\
n_{1} & 0 & 0 & 0 \\
0 & 0 & A_{1}
\end{array}\right) .
$$

having characteristic equation

$$
\left(\lambda^{2}+a_{1} \lambda+a_{2}\right)\left(\lambda+a_{3}\right)\left(\lambda+a_{4}\right)=0,
$$

where

$$
a_{1}=\frac{r\left(m_{2}+n_{2}\right)}{K \beta}>0 ; a_{2}=\beta^{2} x_{1}^{*} x_{2}^{*}>0 ; a_{3}=m_{3}+n_{3}>0 ; a_{4}=m_{4}+n_{4}>0 .
$$

Hence, applying the Routh-Hurwitz criterion, we obtain the following

Theorem 3.1. Under condition (3.4), system (3.1a)-(3.1d) has unique positive equilibrium $x^{*}$, which is asymptotically stable.

The latter theorem, in biological terms, guarantees stable coexistence of the system.

\subsection{Controllability of the system}

Let us introduce time-dependent controls in systems (3.1a)- (3.1d) in two ways. First, we add new parasitized eggs of sugarcane borer. In mathematical terms, this control as a positive effect, is formalized as a time-dependent decrease $u_{2}(t)$ in mortality rate $m_{2}$ of parasitized eggs. Similarly, we apply a selective chemical control to the small larvae of sugarcane borer, and this control, as a negative effect, is considered as a time-dependent increase $u_{3}(t)$ in mortality rate $m_{3}$ of small larvae. Hence we have the following control system.

$$
\begin{aligned}
& \dot{x}_{1}=r x_{1}\left(1-\frac{x_{1}}{K}\right)-m_{1} x_{1}-n_{1} x_{1}-\beta x_{1} x_{2} \\
& \dot{x}_{2}=\beta x_{1} x_{2}-\left(m_{2}-u_{2}(t)\right) x_{2}-n_{2} x_{2} \\
& \dot{x}_{3}=n_{1} x_{1}-\left(m_{3}+u_{3}(t)\right) x_{3}-n_{3} x_{3} \\
& \dot{x}_{4}=n_{3} x_{3}-m_{4} x_{4}-n_{4} x_{4}
\end{aligned}
$$


Remark 3.1. In the above model we have supposed that the applied pesticide kills only small larvae of the plague. In fact, there are pesticides that are effective and applied to a particular cycle of insect pests (Lambert \& Peferoen, 1992; Barrett et al., 2002; Yu, 2008; Dhadialla et al., 2010)

Remark 3.2. We note that from Theorem A.1 of Appendix and from the continuous dependence of the solution on the control, it follows that for controls small enough, the solutions of system (3.7a) - (3.7d) remain in the positive orthant.

Our main objective is a qualitative and quantitative analysis of control system (3.7a) (3.7d), applying the theoretical results of the previous section, concerning nonlinear verticum-type control systems. In the present subsection Theorem 2.2 will be applied to show that our population system can be controlled into equilibrium.

We start with the analysis of the first subsystem

$$
\begin{aligned}
& \dot{x}_{1}=r x_{1}\left(1-\frac{x_{1}}{K}\right)-m_{1} x_{1}-n_{1} x_{1}-\beta x_{1} x_{2} \\
& \dot{x}_{2}=\beta x_{1} x_{2}-\left(m_{2}-u_{2}(t)\right) x_{2}-n_{2} x_{2}
\end{aligned}
$$

With function $F_{0}: R^{3} \rightarrow R^{2}$

$$
F_{0}\left(x_{1}, x_{2}, u_{2}\right):=\left(\begin{array}{c}
r x_{1}\left(1-\frac{x}{K}\right)-m_{1} x_{1}-n_{1} x_{1}-\beta x_{1} x_{2} \\
\beta x_{1} x_{2}-\left(m_{2}-u_{2}\right) x_{2}-n_{2} x_{2}
\end{array}\right),
$$

control system (3.8a)-(3.8b) takes the form

$$
\dot{x}^{1}=F_{0}\left(x^{1}, u_{2}^{*}+u_{2}(t)\right) .
$$

Obviously, to $u_{2}^{*}:=0$ and $u_{2}(t):=0 \quad(t \in[0, T])$, there corresponds the positive equilibrium $x^{1 *}$ of dynamic system (3.1a)-(3.1b).

Now we show that control system (3.8) is locally controllable to $x^{1 *}$ on $[0, T]$. For the application of Theorem A.2 of the Appendix, let us calculate the Jacobians

$$
A_{00}:=\frac{\partial F_{0}}{\partial x^{1}}\left(x^{1 *}, 0\right)=\left[\begin{array}{cc}
-\frac{r}{K} x_{1}^{*} & -\beta x_{1}^{*} \\
\beta x_{2}^{*} & 0
\end{array}\right], B_{0}:=\frac{\partial F_{0}}{\partial u_{2}}\left(x^{1 *}, 0\right)=\left[\begin{array}{c}
0 \\
x_{2}^{*}
\end{array}\right] .
$$

Since

$$
\operatorname{det}\left[B_{0} \mid A_{00} B_{0}\right]=\beta x_{1}^{*}\left(x_{2}^{*}\right)^{2} \neq 0
$$

we get $\operatorname{rank}\left[B_{0} \mid A_{00} B_{0}\right]=2$, and applying Theorem A.2 of Appendix we obtain local controllability of system (3.8) into $x^{1 *}$ on interval $[0, T]$.

Analogously, let us consider the second subsystem

$$
\begin{aligned}
& \dot{x}_{3}=n_{1} x_{1}-\left(m_{3}+u_{3}(t)\right) x_{3}-n_{3} x_{3} \\
& \dot{x}_{4}=n_{3} x_{3}-m_{4} x_{4}-n_{4} x_{4}
\end{aligned}
$$

With notation $F_{1}: R^{4} \rightarrow R^{2}$ 


$$
F_{1}\left(x_{1}, x_{3}, x_{4}, u_{3}\right):=\left(\begin{array}{c}
n_{1} x_{1}-\left(m_{3}+u_{3}\right) x_{3}-n_{3} x_{3} \\
n_{3} x_{3}-m_{4} x_{4}-n_{4} x_{4}
\end{array}\right),
$$

for the control system (3.9a)-(3.9b) we get

$$
\dot{x}^{2}=F_{1}\left(x_{1}, x^{2}, u_{3}^{*}+u_{3}(t)\right) .
$$

Now, to $u_{3}^{*}:=0$ and $u_{3}(t):=0 \quad(t \in[0, T])$, there corresponds the positive equilibrium $\left(x_{1}^{*}, x^{2^{*}}\right)$ of dynamic system (3.1c)-(3.1d).

For local controllability of control system (3.9) to $\left(x_{1}^{*}, x^{2^{*}}\right)$ on $[0, T]$, we calculate the Jacobians

$$
\begin{gathered}
A_{11}:=\frac{\partial F_{1}}{\partial x^{2}}\left(x_{1}^{*}, x^{2^{*}}, 0\right)=\left[\begin{array}{cc}
-m_{3}-n_{3} & 0 \\
n_{3} & -m_{4}-n_{4}
\end{array}\right], \\
A_{10}:=\frac{\partial F_{1}}{\partial x_{1}}\left(x_{1}^{*}, x^{2^{*}}, 0\right)=\left[\begin{array}{c}
n_{1} \\
0
\end{array}\right] B_{1}:=\frac{\partial F_{1}}{\partial u_{3}}\left(x_{1}^{*}, x^{2 *}, 0\right)=\left[\begin{array}{c}
-x_{3}^{*} \\
0
\end{array}\right] .
\end{gathered}
$$

From

$$
\operatorname{det}\left[B_{1} \mid A_{11} B_{1}\right]=n_{3}\left(x_{3}^{*}\right)^{2} \neq 0 \text {, }
$$

again we get $\operatorname{rank}\left[B_{1} \mid A_{11} B_{1}\right]=2$, and applying Theorem A.2 we obtain the local controllability of system (3.9) into $x^{2 *}$ on interval $[0, T]$.

Then by Theorem 2.2, we obtain the following.

Theorem 3.2. Control system (3.7a)-(3.7d) is controllable near its equilibrium $x^{*}$.

\subsection{Calculation of an equilibrium control}

Fix an initial state $x_{0}$ from a neighbourhood of local controllability of system (3.7), and for each control function $u$ small enough (i.e. $u \in U_{\varepsilon}[0, T]$ for appropriate $\left.\left.\varepsilon \in\right] 0, \varepsilon_{0}\right]$, see conditions of system (A.1)-(A.2) of Appendix), let $x$ be the solution of (3.7) defined on $[0, T]$ and corresponding to the initial value $x_{0}$. Then a control $u \in U_{\varepsilon}[0, T]$ will steer initial state $x_{0}$ into equilibrium $x^{*}$, if and only if it minimizes functional

$$
\Phi(u):=\left|x(T)-x^{*}\right|^{2}\left(u \in U_{\varepsilon}[0, T]\right) .
$$

The above reasoning can be summarized in the following theorem:

Theorem 3.3. Suppose that the parameters of system (3.7) satisfy conditions (3.4). Then system (3.7) is locally controllable to equilibrium $x^{*}$ on interval $[0, T]$. An initial state $x_{0}$ from a neighbourhood of local controllability will be steered into $x^{*}$ by a control $u \in U_{\varepsilon}[0, T]$ if and only if the latter is a solution of the following optimal control problem:

$$
\Phi(u):=\left|x(T)-x^{*}\right|^{2} \rightarrow \min ,
$$




$$
\begin{gathered}
u \in U_{\varepsilon}[0, T], x(0)=x_{0}, \\
\dot{x}=F\left(x, u^{*}+u(t)\right) .
\end{gathered}
$$

Example 3.1 With the parameters of Rafikov and Limeira (2012), let us consider system (3.1) with parameters

$n_{1}=n_{2}=0.1 ; \quad n_{3}=n_{4}=0.0244 ; \quad m_{1}=m_{2}=0.036 ; \quad m_{3}=0.001 ; \quad m_{4}=0.0016 \quad$ and $K=25000$.

On the other hand, choosing $r=0.19008 ; \beta=0.0003$, condition (3.4) is verified.

Then, for these parameters we have the following positive equilibrium of model (3.1):

$$
x_{1}^{*}=452.8 ; x_{2}^{*}=170.8 ; x_{3}^{*}=1784 ; x_{4}^{*}=1675 \text {. }
$$

The main objective of the biological pest control is to maintain the pest population in an equilibrium level below the economic level. For this pest density the threshold level is 2500 (see Rafikov and Limeira (2012)).

For system (3.1), with initial condition $x^{0}:=(100,200,2500,500)$, the corresponding solution $x$, tending to equilibrium $x^{*}=\left(x_{1}^{*}, x_{2}^{*}, x_{3}^{*}, x_{4}^{*}\right)$, can be seen in Figure 1.

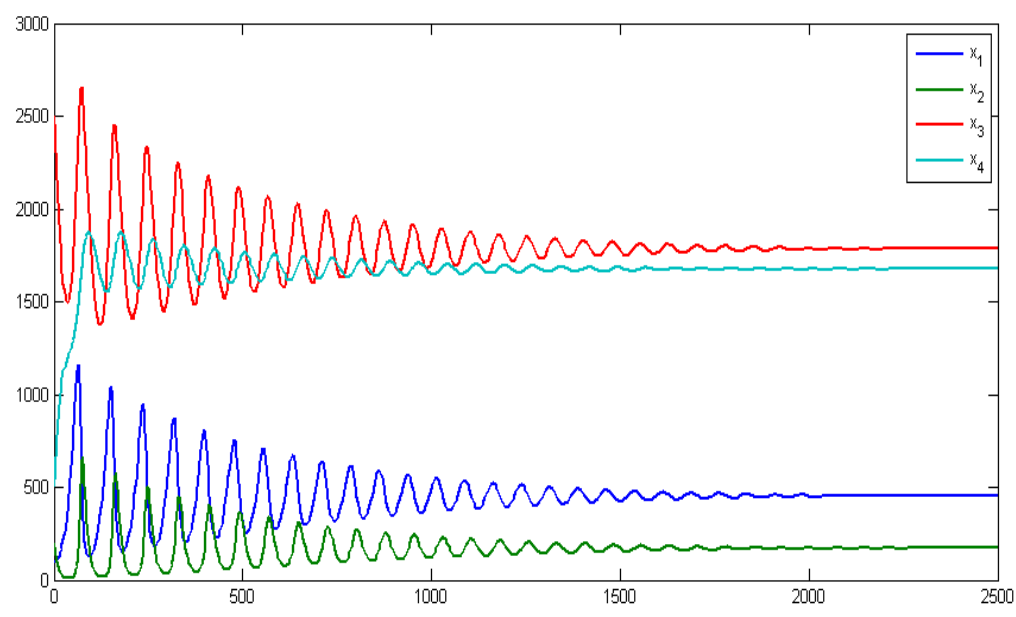

Figure 1. Solution of system (3.1), with initial value $x^{0}:=(100,200,2500,500)$

Applying the results found in the previous sections, our objective is to determine a control of system (3.7), that steers the system into equilibrium.

Fix time duration $T:=200$ and take initial condition $x^{01}:=(100,200)$ for system $(3.8)$. For the calculation of the corresponding solution we apply the MatLab toolbox mentioned above. Figure 2.b) shows the obtained optimal control $u_{2}(t)$; the corresponding solution $x^{1}$ ending up at equilibrium $x^{1 *}=(4.85,3.12)$ can be seen in Figure 2.a). 


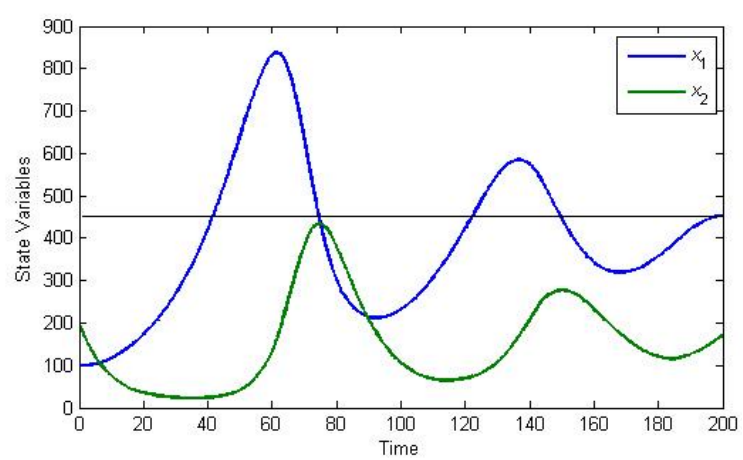

a)

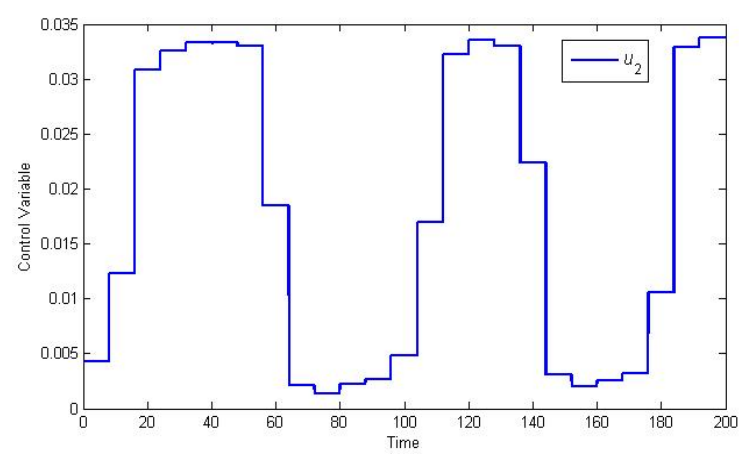

b)

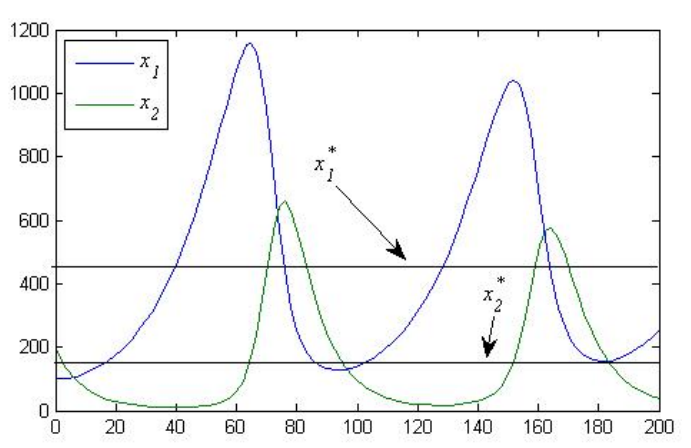

c)

Figure 2. a) Solution of control system (3.8) for $T=200$, with initial value $x^{01}:=(100,200)$, b) Control function of system (3.8) for $T=200$, c) Solution of the uncontrolled system (3.5) for $T=200$, with initial value $x^{01}:=(100,200)$.

With $T:=200$ and initial condition $x^{02}:=(2500,500)$ for system $(3.9)$, we calculate the solution. Figure 3.b) shows the obtained optimal control $u_{3}$; the corresponding solution $x^{2}$ ending up at equilibrium $x^{2 *}=(1784,1675)$ can be seen in Figure 3.a).

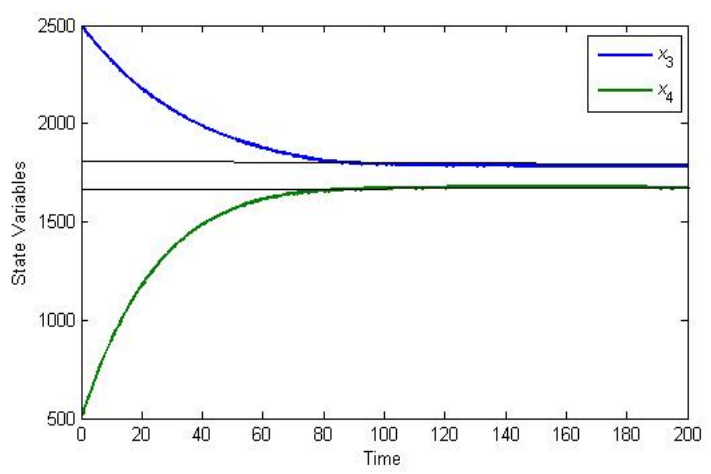

a)

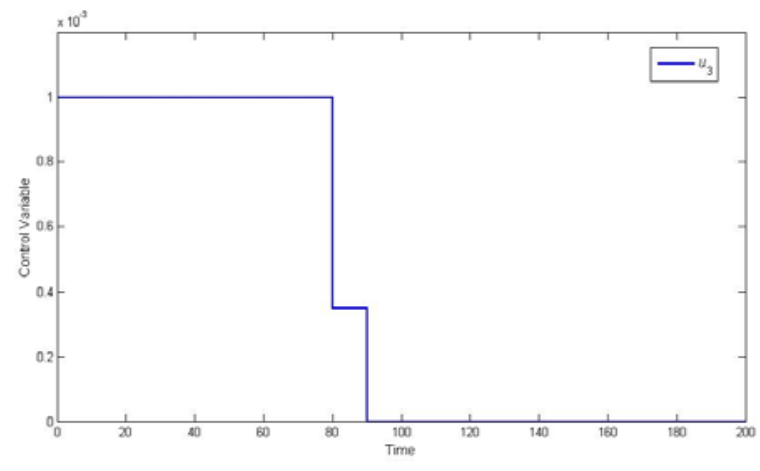

b) 


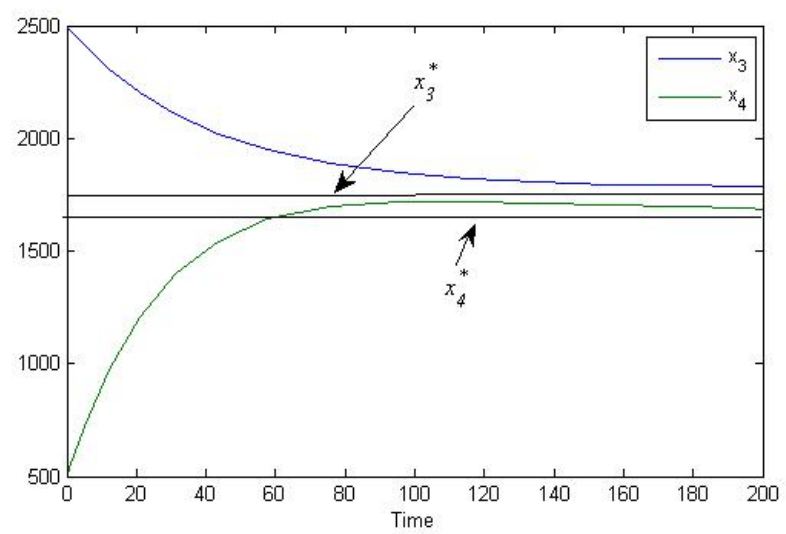

c)

Figure 3. a) Solution of control system (3.9) for $T=200$, with initial value $x^{02}:=(2500,500)$, b) Control function of system (3.9) for $T=200$, c) Solution of the uncontrolled system (3.6) for $T=200$, with initial value $x^{02}:=(2500,500)$.

Finally, if for system (3.7) we take initial condition $x^{0}=\left(x^{01}, x^{02}\right)$, with the values of the two previous examples, we can check that with the obtained controls the system arrives into the equilibrium in time $T=200$ (see Figure 4).

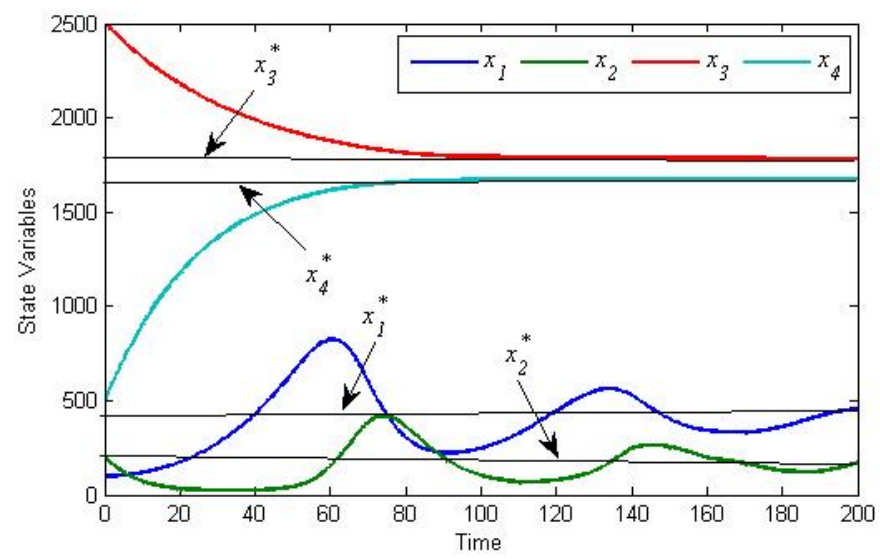

Figure 4. Solution of control system (3.7) for $T=200$, with initial value $x^{0}=(100,200,2500,500)$.

It is easy to see that the control that steers the system into the equilibrium is not unique, we can optimize over the set of equilibrium controls, minimizing the total cost. This problem will be addressed in the next section.

\subsection{Optimal equilibrium control}

For a more flexible model, we will consider the corresponding integral with and without discount. Although for infinite time-horizon problems an exponential discount factor is a technical necessity (see Clark, 2010 and references therein), similar discount is also used in finite time-horizon models (see e.g. Chakraborty et al. 2011).

For $\varepsilon>0$ of the previous subsection, with the same controlled population dynamics as (3.7), the corresponding optimal control problem is the following: 


$$
\begin{gathered}
\Psi(u):=\int_{0}^{T} c_{2} u_{2}(t)+c_{3} u_{3}(t) d t \rightarrow \min , \\
u \in U_{\varepsilon}[0, T] \\
\dot{x}=F\left(x, u^{*}+u(t)\right), \\
x(0)=x_{0}, \quad x(T)=x^{*} .
\end{gathered}
$$

Now, for a numerical solution of this problem using the mentioned MatLab toolbox of Banga et al. (2005), piecewise constant controls are considered. More precisely, for fixed positive integer $N$, let $t_{i}:=i \frac{T}{N} \quad(i \in \overline{0, N})$ be the uniform division of $[0, T]$, and let us define the set of controls as follows:

$S_{\varepsilon, N}[0, T]:=\left\{u \in U_{\varepsilon}[0, T]: u\right.$ is constant on each interval $] t_{i-1}, t_{i}[(i \in \overline{1, N})\}$.

Then, considering the set of admissible controls

$$
S_{\varepsilon, N}^{*}[0, T]:=\left\{u \in S_{\varepsilon, N}[0, T]: u \text { satisfies (3.13) and (3.14) }\right\} \text {, }
$$

$\varepsilon>0$ and $N$ are chosen as to guarantee that $S_{\varepsilon, N}^{*}[0, T]$ is not empty. Hence functional $\Psi$ in (3.12) can be defined on the compact set $S_{\varepsilon, N}^{*}[0, T] \subset \mathrm{R}^{N}$ and is the composition of two continuous mappings $S_{\varepsilon, N}^{*}[0, T] \rightarrow S_{\varepsilon, N}^{*}[0, T] \times C[0, T]$, assigning to each $u \in S_{\varepsilon, N}^{*}[0, T]$ the pair $(u, x)$, where $x$ is the solution of (3.14), corresponding to $u$, and mapping $S_{\varepsilon, N}^{*}[0, T] \times C[0, T] \rightarrow \mathrm{R}$, assigning to each pair

$(u, x) \in S_{\varepsilon, N}^{*}[0, T] \times C[0, T]$ the integral $\Psi(u)=\int_{0}^{T} c_{2} u_{2}(t)+c_{3} u_{3}(t) d t$. As a result of the above reasoning, we obtain the following

Theorem 3.4. For any parameter choice satisfying conditions (3.4), the optimal control problem

$$
\begin{gathered}
\Psi(u):=\int_{0}^{T} c_{2} u_{2}(t)+c_{3} u_{3}(t) d t \rightarrow \min , \\
u \in S_{\varepsilon, N}^{*}[0, T] \\
\dot{x}=F\left(x, u^{*}+u(t)\right) \\
x(0)=x_{0}, \quad x(T)=x^{*}
\end{gathered}
$$

has a solution.

The obtained result shows a possible bargain between the bioeconomic and integrated control. Now we proceed to the illustration of the above optimal control model for different discount parameter values.

Example 3.2. For the same parameters used in the previous example (Example 3.1) for model (3.7), we take $c_{2}=10 ; c_{3}=0.001$ for the constant weights in the cost functional 
$\Psi$. In Figure 5.a) we see how the trajectories arrive at the equilibrium, minimizing the given functional with the controls shown in Figure 5.b). We remind that these controls act in different phases of development, therefore when the level of larvae is low enough (after time 140), the chemical control is reduced and even stopped (at time 150), and the application of biological control acting directly on eggs is sufficient. This fact is also very reasonable, since in this way the appropriate chemical agent may have time to decompose.

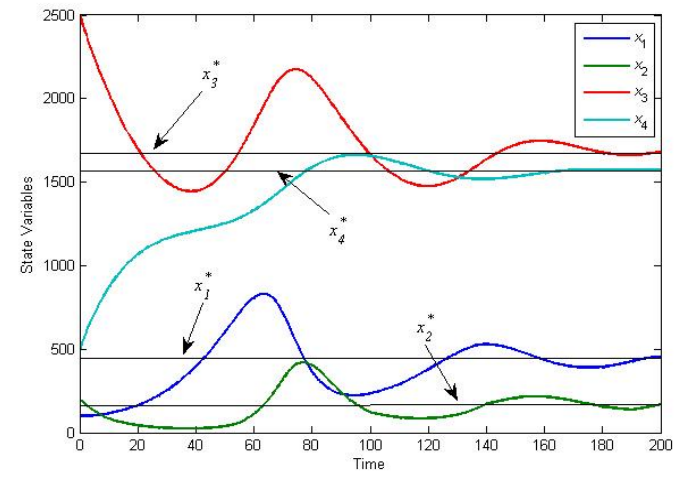

a)

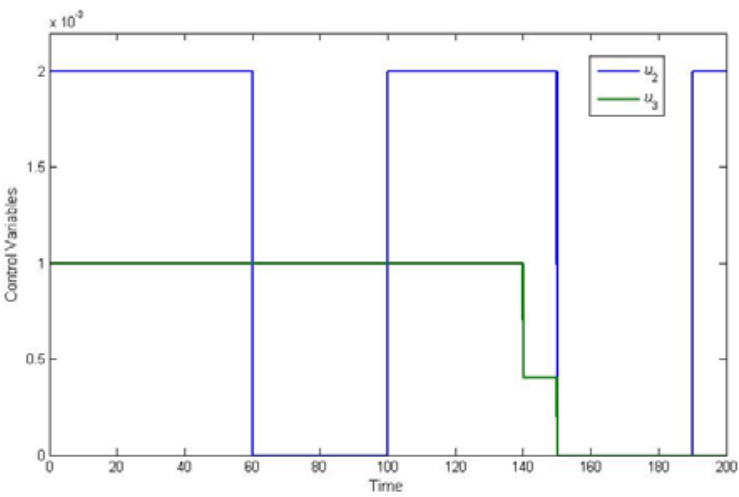

b)

Figure 5. a) Solution of control system (3.7) for $T=200$, with initial value $x_{0}=(100,200,2500,500)$. b) Control function of system (3.7) for $T=200$.

\section{Discussion}

We have extended earlier results on controllability of linear verticum-type systems to the nonlinear case, giving a sufficient condition for local controllability of nonlinear verticum-type systems. We note that although the given rank condition is only sufficient but not necessary, this fact does not limit the possibilities of applications.

The study of the nonlinear case was mainly motivated by the fact that most models of population ecology are nonlinear. Our general theorem is applied to the study of an entomo-ecological model of integrated pest control where, apart from a parasitoid agent a chemical control is also applied. We have seen that under certain conditions, the uncontrolled system tends to an equilibrium where, however, the density of the pest may be too high. Our control model makes it possible not only to read an equilibrium more quickly, but also steer the system to an equilibrium in given time, where the pest density is below a given "economic threshold". The latter means that after the damage caused by the pest, it is still economically reasonable to maintain the crops.

At this point we remark that our model can be modified in two alternative ways to use a predator agent instead of chemical control. One of the possibilities is to consider the control function as the action of a specific predator of small larvae, adding an equation to the second subsystem that describes predator dynamics. The other option is introducing an additive control term (instead of the multiplicative control representing the chemical agent) describing the time-dependent release of predator agents. In this case there is no need to include a predator dynamics in the system. 


\section{Acknowledgements}

The present research has been supported by the Hungarian Scientific Research Fund OTKA (K81279). Excellence Project Programme of the Ministry of Economy, Innovation and Science of the Andalusian Regional Government, and was also funded by FEDER Funds under reference: P09-AGR-5000

\section{References}

Banga, J.R., Balsa-Canto, E., Moles, C.G., Alonso, A.A., (2005). Dynamic optimization of bioprocesses: efficient and robust numerical strategies. Journal of Biotechnology 117, 407-419.

Barrett, J. W.; Primavera, M.; Retnakaran, A.; Arif, B.; Palli, S. R., (2002). Aspects of nucleopolyhedrovirus pathogenesis in lepidopteran larvae, pp.: 205-214. In: Koul, O.; Dhaliwal, G. S. (eds.). Microbial biopesticides. Taylor \& Francis Inc. New York, NY, USA.

Chen, B.M., Lin, Z., Shamesh, Y. (2004). Linear Systems Theory. A Structural Decomposition Approach, Birkhauser, Boston.

Clark, C.W. (2010). Mathematical Bioeconomics: The Mathematics of Conservation. John Wiley \& Sons.

Dhadialla, T. S.; Retnakaran, A. and Smagghe, G., (2010). Insect growth- and development-disrupting insecticides, pp.: 121-181. In: Gilbert, L.I. and Gill, S.S. (eds.). Insect control: Biological and synthetic agents. Academic Press, London, UK.

Gámez M. (2011). Observation and Control in Density- and Frequency-dependent Population Models. In: WenJun Zhang (Ed.), Ecological Modeling. Nova Science Publishers, New York, pp. 285-306.

Gámez, M., López, I., Garay, J. and Varga, Z. (2009). Observation and control in a model of a cell population affected by radiation. Biosystems 96 (2009) 172-177.

Gámez, M. López, I., Szabó, I. and Varga, Z. (2010). Verticum-type systems applied to ecological monitoring. Applied Mathematics and Computation, 215, 3230-3238.

Gámez, M., López, I., Garay, J. and Varga, Z. (2011). Monitoring and control in a spatially structured population model. In: B. Murgante, O. Gervasi, A. Iglesias, D. Taniar, B. O. Apduhan (Eds.), Computational Science and Its Applications Vol. V, pp. 511-520, "Lecture Notes in Computer Science" 6786, Springer-Verlag Berlin Heidelberg.

Gámez, M., López, I., Varga Z. and Garay, J. (2012). Stock estimation, environmental monitoring and equilibrium control of a fish population with reserve area. Reviews in fish biology and fisheries, 22 (3), 751-766

Lee, E.B.; Markus, L. (1971). Foundations of Optimal Control Theory. Wiley, New York-London-Sydney 
López, I., Gámez, M. and Carreño, R. (2004). Observability in dynamic evolutionary models. Biosystems, 73, 99-109.

López I, Gámez M, Molnár, S. (2007a). Observability and observers in a food web. Applied Mathematics Letters 20 (8): 951-957.

López, I., Gámez, M., Garay, J. and Varga, Z. (2007b). Monitoring in a Lotka-Volterra model. BioSystems Vol. 87, No. 1, 68-74

Molnár, S. (1989). A special decomposition of linear systems. Belgian Journal of Operations Research, Statistics and Computer Science, 29 (4): 1-19.

Molnár, S. (1993). Stabilization of verticum-type systems, Pure Mathematics and Applications, 4 (4): 493-499.

Molnár, S., Gámez, M. and López, I. (2012). Observation of nonlinear verticum-type systems applied to ecological monitoring. International Journal of Biomathematics. DOI No: 10.1142/S1793524512500519

Molnár, S., Szigeti, F. (1994). On "Verticum"-Type Linear Systems with TimeDependent Linkage. Applied Mathematics and Computation 60: 89-102.

Rafikov, M., Limeira, E.H. (2012). Mathematical Modelling of the Biological Pest Control of the Sugarcane Borer, International Journal of Computer Mathematics, Vol 89 No 3, 390-401.

Scarelli, A., Varga, Z. (2002). Controllability of selection-mutation systems. BioSystems. 65, No 2-3, 113-121.

Shamandy, A. (2005). Monitoring of trophic chains. Biosystems, Vol. 81, No. 1, 43-48.

Varga, Z. (1989). On controllability of Fisher's model of selection. In "Differential Equations" (Eds. C. M. Dafermos, G. Ladas, G. Papanicolau) Marcel Dekker, New York, 717-723.

Varga, Z. (1992). On Observability of Fisher's model of selection. Pure Mathematics and Applications, Ser. B. Vol. 3 No 1, 15-25.

Varga, Z., Scarelli, A. and Shamandy, A. (2002). An observability problem of population ecology. In: V. Capasso (Ed.), Mathematical Modelling and Computing in Biology and Medicine, 5th ECMTB Conference 2002 (Abstracts, p.189). MIRIAM, Milan, Italy.

Varga, Z., Scarelli, A., Shamandy, A. (2003). State monitoring of a population system in changing environment. Community Ecology, 4, (1) 73-78.

Varga, Z. (2008a). Observer Design for Genetic Processes. Mechanical Engineering Letters Vol. 1, 13-25.

Varga, Z. (2008b). Applications of mathematical systems theory in population biology. Periodica Mathematica Hungarica , Vol. 56 (1), 157-168.

Varga, Z., Gámez, M. and López, I. (2010). Observer design for open and closed trophic chains. Nonlinear Analysis: Real World Applications 11, pp. 1918-1924.

Yu, S.J., (2008). The toxicology and biochemistry of insecticides. CRC Press. Boca Raton, FL, USA: 276 pp. 


\section{Appendix}

Given $n, r \in N$, let $F: R^{n} \times R^{r} \rightarrow R^{n}$ be a continuously differentiable function. For a reference control value $u^{*} \in R^{r}$, let $x^{*} \in R^{n}$ be such that $F\left(x^{*}, u^{*}\right)=0$. For technical reason we shall need a rather general class of controls. Let us fix a time interval $[0, T]$, and for each $\varepsilon \in R^{+}$define the class of essentially bounded $\varepsilon$-small controls

$$
U_{\varepsilon}[0, T]:=\left\{u \in L_{\infty}^{r}[0, T] \mid\|u(t)\|_{\infty} \leq \varepsilon \text { for almost every } t \in[0, T]\right\} .
$$

From Lee and Markus (1971) we recall the following

Theorem A.1. There exists $\varepsilon_{0} \in R^{+}$such that for all $u \in U_{\varepsilon_{0}}[0, T]$ and $x^{0} \in R^{n}$ with $\left\|x^{0}-x^{*}\right\|<\varepsilon_{0}$ the initial value problem

$$
\begin{aligned}
& \dot{x}(t)=F\left(x(t), u^{*}+u(t)\right) \quad(\text { for a.e. } t \in[0, T]) \\
& x(0)=x^{0}
\end{aligned}
$$

has a unique solution. We notice that $x^{*}$ is an equilibrium state for the zero-control system.

Definition A.1. Control system (A.1)-(A.2) is said to be locally controllable to $x^{*}$ on $[0, T]$, if there exists $\left.\varepsilon \in] 0, \varepsilon_{0}\right]$ such that for all $x^{0}$ from the $\varepsilon$-neighbourhood of $x^{*}$, there is a control $u \in U_{\varepsilon}[0, T]$ that controls the initial state $x^{0}$ to equilibrium $x^{*}$, i.e. for the solution $x$ of the initial value problem (A.1)-(A.2), equality $x(T)=x^{*}$ holds.

Let us linearize system (A.1)-(A.2) around $\left(x^{*}, u^{*}\right)$, introducing the corresponding Jacobians

$$
A:=\frac{\partial}{\partial x} F\left(x^{*}, u^{*}\right), \quad B:=\frac{\partial}{\partial u} F\left(x^{*}, u^{*}\right) .
$$

Then we have the following sufficient condition for local controllability:

Theorem A.2 (Lee and Markus, 1971)

If

$$
\operatorname{rank}\left[\begin{array}{c}
B \\
A B \\
\vdots \\
A^{n-1} B
\end{array}\right]=n
$$

then system (A.1)-(A.2) is locally controllable to $x^{*}$ on $[0, T]$. 